\title{
The Association between Age-Related Macular Degeneration and the Risk of Mortality
}

\author{
Peipei Wang, ${ }^{1,2}$ Jie Wang, ${ }^{1}$ Jun Ma, ${ }^{1}$ Ge Jin, ${ }^{1}$ and Xueqiang Guan ${ }^{1}$ \\ ${ }^{1}$ Department of Cardiology, The Second Affiliated Hospital and Yuying Children's Hospital, Wenzhou Medical University, \\ Xueyuanxi Road, No. 109, Wenzhou, Zhejiang 325000, China \\ ${ }^{2}$ Department of Neurology, Wenzhou City Hospital of Traditional Chinese Medicine and Western Medicine Combined, \\ Wenzhou, China \\ Correspondence should be addressed to Xueqiang Guan; wzsgxq@163.com
}

Received 6 March 2017; Revised 23 April 2017; Accepted 27 April 2017; Published 18 May 2017

Academic Editor: Alfredo García-Layana

Copyright (C) 2017 Peipei Wang et al. This is an open access article distributed under the Creative Commons Attribution License, which permits unrestricted use, distribution, and reproduction in any medium, provided the original work is properly cited.

\begin{abstract}
Studies have investigated the association between age-related macular degeneration (AMD) and subsequent risks of mortality, but results have been equivocal. We conducted a comprehensive analysis of prospective cohort studies to assess the association of AMD and the risk of mortality in the general population. We searched PubMed and EMBASE for trials published from 1980 to 2016 . We included 11 cohort studies that reported relative risks with 95\% confidence intervals for the association of AMD and mortality, involving 57,069 participants. In a random-effects model, the adjusted RR (95\% confidence interval) associated with AMD was 1.09 (1.02-1.17) for all-cause mortality. Findings from this research provide support that persons with AMD had a higher subsequent risk of mortality than persons without AMD.
\end{abstract}

\section{Introduction}

Age-related macular degeneration (AMD) is a progressive blinding disease in adults over 50 years of age [1-3]. It is estimated to affect approximately $8.7 \%$ of the worldwide population, and the number is anticipated to reach 288 million by the year $2040[1,2]$. This results in an annual $\$ 4.6$ billion direct healthcare cost in the United States [4]. As the aging population increases, AMD is becoming a global public health crisis [1]. AMD has both early and late stages [5]. It is affected by the dysfunction of a specialized cell layer in the back of the eye called the retinal pigmented epithelium $[1,5]$. Early AMD is usually not associated with loss of vision [6]. And late AMD is manifested through geographic atrophy or the development of neovascularization $[4,6]$. Neovascular or wet AMD leads to AMD-related visual loss $[1,6]$.

Several studies [7-18] have investigated the association between AMD and subsequent risks of mortality, but results have been equivocal. A previous pooling analysis [19] by Wang et al. that included 20 cohort studies that focused on cardiovascular outcomes and all-cause mortality suggested that AMD is associated with an increased risk of all-cause mortality. However, the review omitted many important papers which were eligible and did not investigate the AMD and mortality association according to study characteristics.

Therefore, we conducted a comprehensive meta-analysis of prospective cohort studies to assess the association of AMD and the risk of mortality in the general population.

\section{Methods}

2.1. Search Strategy. We conducted a PubMed and EMBASE search through February 2015 for studies describing the association between AMD and the risk of mortality. To make sure our study was based on up-to-date results, we further updated the literature search of PubMed and EMBASE in November 2016. Only papers published in peer-reviewed journals and in English language were considered. In addition, additional studies were identified through the reference lists of relevant publications and relevant reviews. We used search terms "Age-related macular degeneration", "AMD", "retina macula degeneration", "retinal degeneration", "mortality" 
and "death" and so on. No attempt was made to identify unpublished reports.

2.2. Study Selection. The investigators (P. Wang and J. Ma) independently assessed article eligibility. Any discrepancies regarding eligibility were resolved by consensus. Studies were eligible for our analysis if (1) the authors reported data from an original, peer-reviewed study (i.e., not review articles, letters, comments, or conference abstracts); (2) the main exposure was AMD; (3) the outcome of interest was allcause mortality; (4) the study was of a prospective cohort design; and (5) relative risk (RR) with corresponding 95\% confidence intervals (CIs) was reported in the article. A study must meet all the five inclusion criteria for inclusion. In the case of multiple publications, we chose the articles with the largest sample or the longest follow-up interval. Studies reporting crude associations without any adjustment were also excluded.

The agreement between the two investigators was $99.3 \%$ for the first screen and $100 \%$ for the full-text articles.

2.3. Data Extraction. The standardized, predefined data was extracted from the studies: last name of the first author, publication year, study location, follow-up years, number of cases and participants, mean baseline age, adjustment covariate, and effect size. If the data was not clear from the studies, we corresponded with the author(s) for the relevant data.

2.4. Data Synthesis and Analysis. The RR was used estimating association of AMD and the risk of all-cause mortality, and the odds ratio or hazard ratio was considered equivalent to the RR [20]. Forest plots were used to visually assess the RR and corresponding 95\% confidence interval across studies. Homogeneity of RR across studies was tested by the Cochrane $Q$ statistic (significance level: $P<0.10$ ) and the $I^{2}$ statistic (ranges from $0 \%$ to $100 \%$ ) [21]. The RR were pooled using the random-effects DerSimonian and Laird models [22]. The possibility of publication bias was evaluated using the visual inspection of a funnel plot [23]. Moreover, subgroup analyses were conducted to evaluate the influences of the selected study and participant characteristics (including regions, case numbers, and types of AMD and number of prescriptions and follow-up year) on the results.

Analyses were performed with the Review Manager software (version 5.2; the Nordic Cochrane Centre, Copenhagen, Denmark). A two-sided $P<0.05$ was considered statistically significant.

\section{Results}

3.1. Literature Search. Figure 1 shows the literature search flow chart. Our search strategy found 360 articles. After the first round of screening based on titles and abstracts with the aforementioned criteria, 20 articles were selected. Subsequently, after detailed examination, 9 literatures were excluded (reasons shown in Figure 1). No study was retrieved

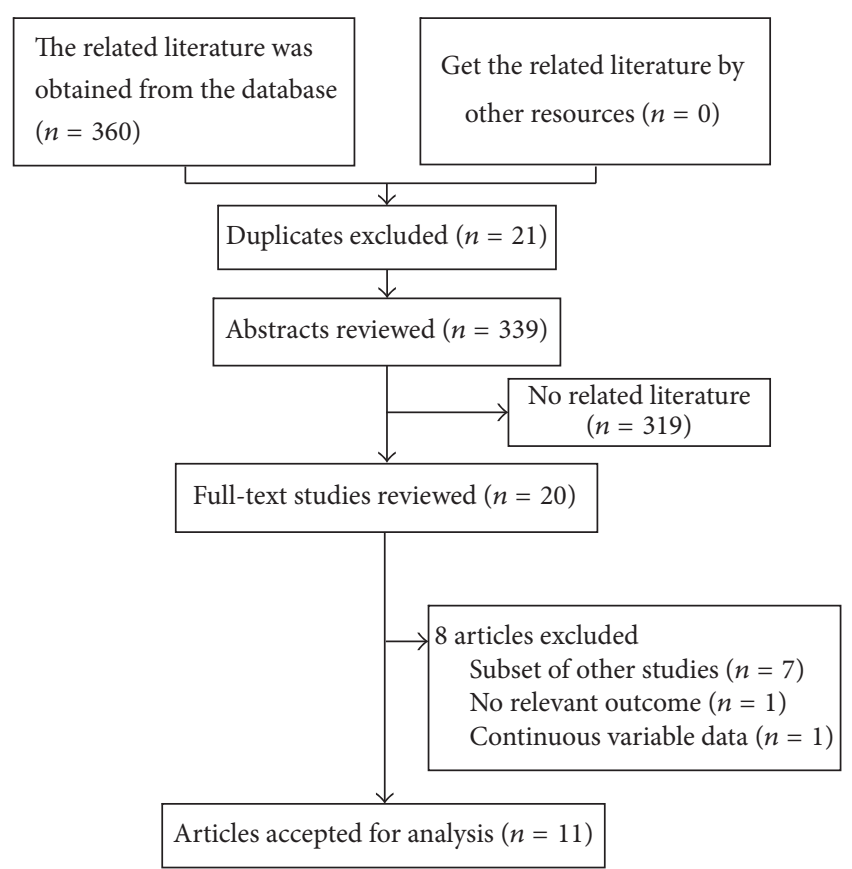

FIGURE 1: Flow chart of the meta-analysis of AMD and mortality.

from the reference lists of relevant articles and reviews. As a result, a total of 11 studies were selected for this meta-analysis.

3.2. Study Characteristics. The characteristics for the 11 included cohort studies are presented in Table 1 . The 11 studies were published between 2001 and 2016. With regard to the study region, two studies were conducted in North America, two in Oceania, three in Asia, and four in Europe. Followup duration ranged from 5 to 15 years, with a median of 7.6 years. The sizes of cases ranged from 32 to 1,341 , with a sum of 5,213. The sizes of participants ranged from 866 to 13,569 with a sum of 57,069. Most studies included both men and women, and only one study [14] was conducted exclusively in women. AMD ascertainments differed between studies, with most using medical records and some using self-report. Adjustment for potential confounding factors also differed between studies, and most risk estimates were adjusted for age, gender, and body mass index.

3.3. Main Analysis. Among 11 studies, the majority of studies showed positive association (i.e., RR > 1.00) between AMD and the risk of mortality, and only two studies reported $\mathrm{RR}<$ 1.00 but not statistically significant. The pooled multivariableadjusted RR (95\% CI) was 1.09 (1.02-1.17; Figure 2), with moderate heterogeneity detected among studies $\left(I^{2}=17 \%\right.$; $P_{\text {heterogeneity }}=0.28$ ).

We conducted stratified analyses by geographic area, number of AMD, and type of AMD. The results of the stratified analysis are shown in Table 2, and the result of subgroup analysis by types of AMD is presented in Figure 3. 


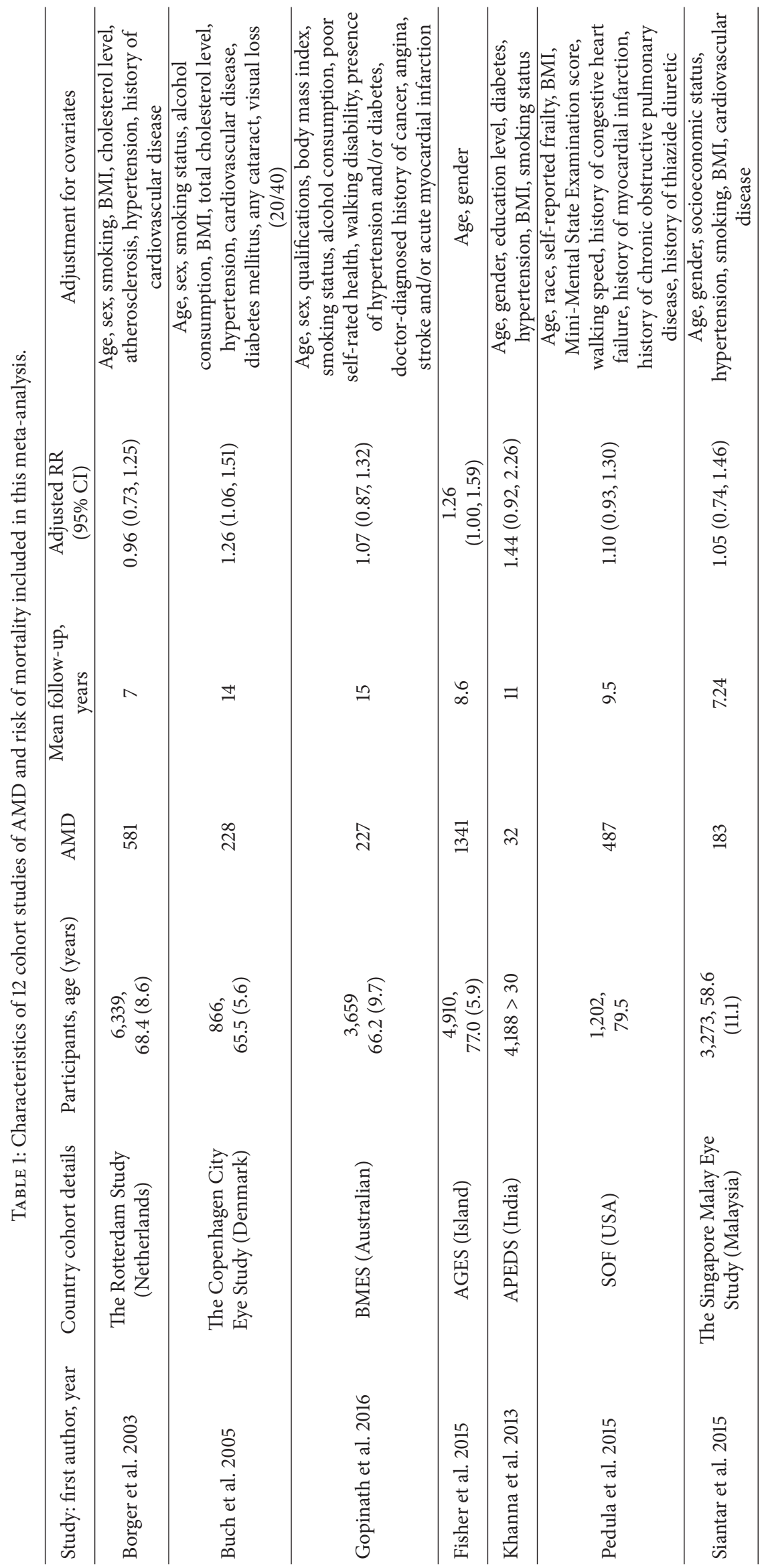




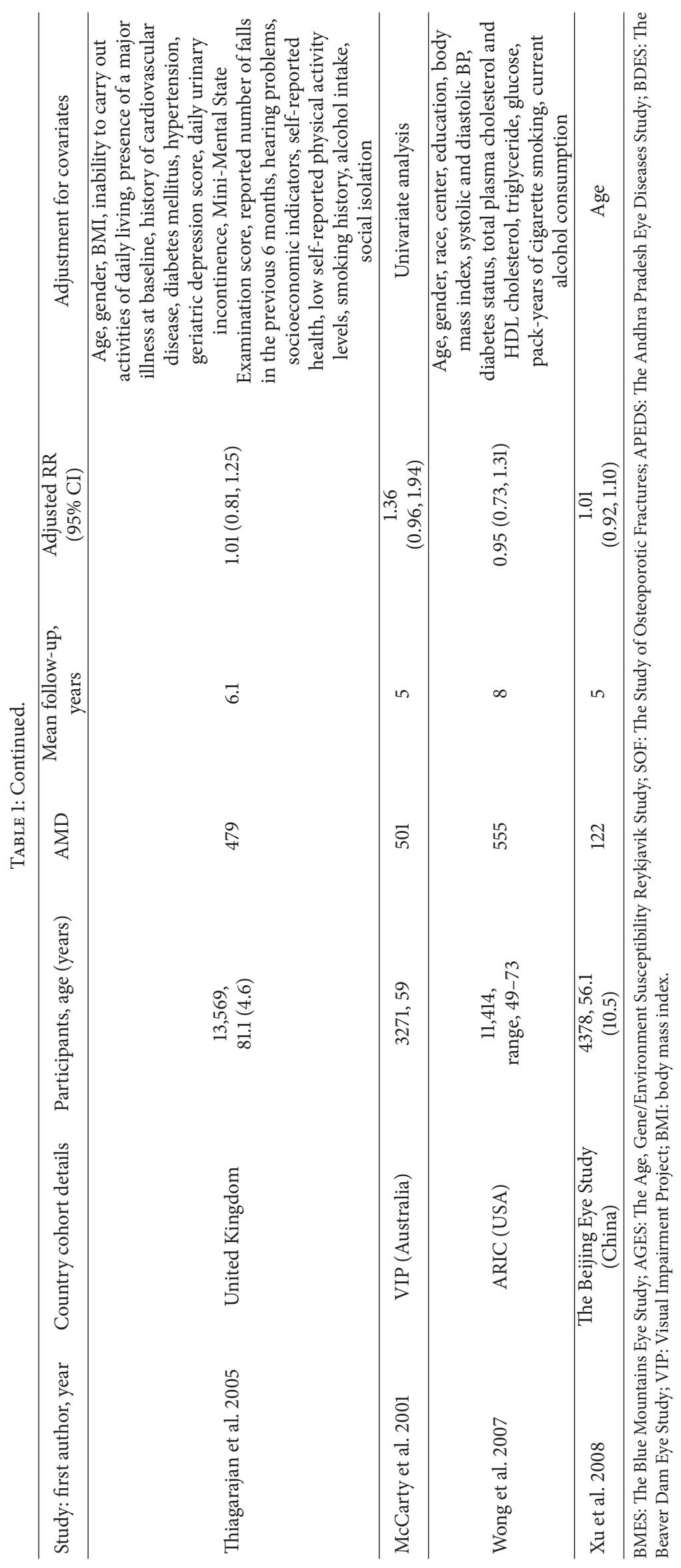




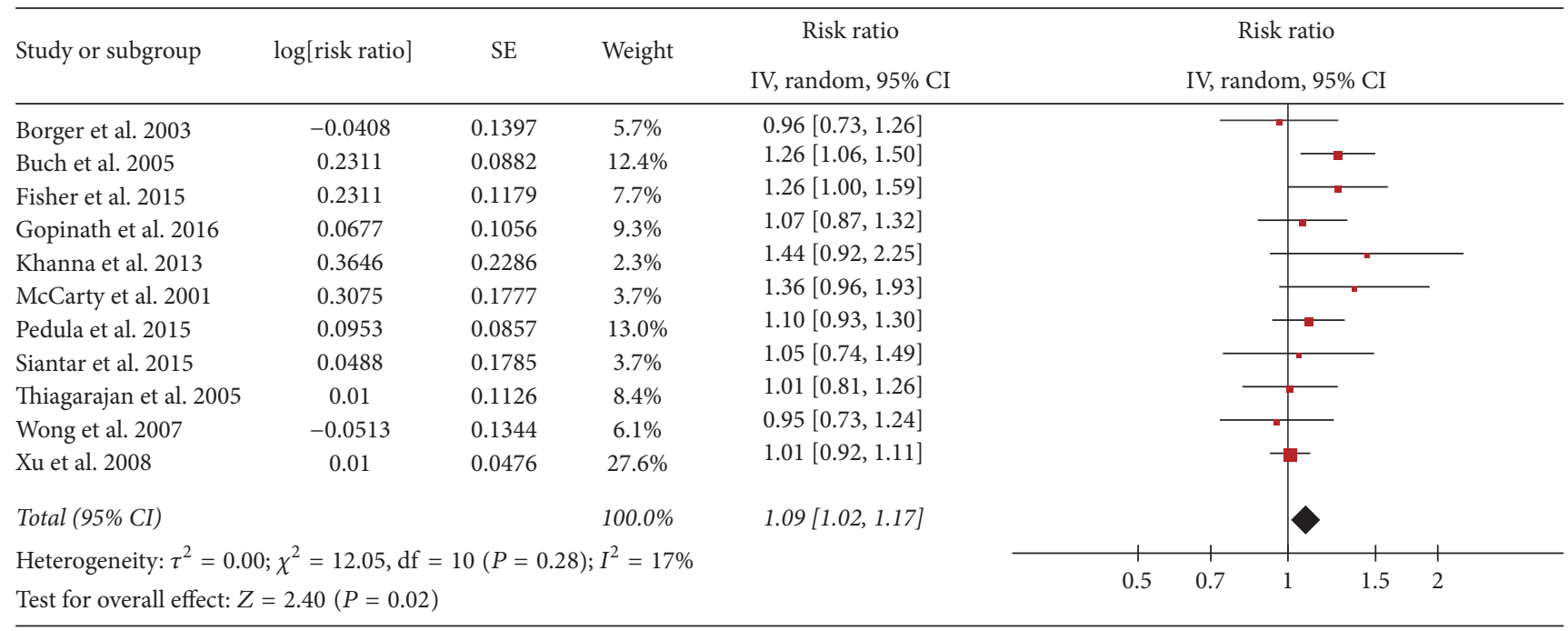

FIGURE 2: Adjusted relative risks of all-cause mortality associated with AMD.

TABLE 2: Stratified analyses of mortality associated with AMD.

\begin{tabular}{|c|c|c|c|c|}
\hline Group & $\begin{array}{c}\text { Number of } \\
\text { studies }\end{array}$ & RR (95\% CI) & $P$ (heterogeneity) & $I^{2}(\%)$ \\
\hline Total & 11 & $1.09[1.02,1.17]$ & 0.28 & 17 \\
\hline \multicolumn{5}{|l|}{ Geographic area } \\
\hline Oceania & 2 & $1.16[0.93,1.14]$ & 0.25 & 26 \\
\hline North America & 2 & $1.05[0.92,1.21]$ & 0.36 & 0 \\
\hline Asia & 3 & $1.04[0.92,1.19]$ & 0.31 & 14 \\
\hline Europe & 4 & $1.13[0.99,1.30]$ & 0.20 & 36 \\
\hline \multicolumn{5}{|l|}{ Number of AMD } \\
\hline$<500$ & 7 & $1.08[1.00,1.16]$ & 0.32 & 14 \\
\hline$\geq 500$ & 4 & $1.11[0.93,1.32]$ & 0.18 & 39 \\
\hline \multicolumn{5}{|l|}{ Type } \\
\hline Early AMD & 8 & $1.06[1.00,1.13]$ & 0.65 & 0 \\
\hline Late AMD & 8 & $1.11[0.96,1.29]$ & 0.07 & 46 \\
\hline
\end{tabular}

3.4. Publication Bias Assessment. The funnel plot for the detection of public bias among studies that evaluated the associations of AMD with the risk of mortality is shown in Figure 4 . The funnel plot was fairly symmetric, indicating that publication bias was not significant.

\section{Discussion}

Our meta-analysis of 11 cohort studies demonstrated significant associations between AMD and all-cause mortality.

For the relationship between AMD and the risk of allcause mortality, the precise mechanism is not clear. Nevertheless, several possible pathogenic mechanisms have been proposed. AMD could be a marker of underlying serious somatic factors or diseases and reflect the status of systemic processes associated with biological aging, which could be associated with decreased survival and increased biological aging [24]. AMD is a chronic disease of the central retina and is a leading cause of low vision among older adults [2]. Low vision reflects functional status and leads to functional problems, such as accidents, falls, fractures, loss of independence, and depression, all of which may be life-threatening [24-26].

The result was consistent with a previous meta-analysis [19] conducted by Wang et al. suggesting a significant association between AMD and all-cause mortality. However, this review focused on cardiovascular outcomes and cardiovascular disease mortality, and many important papers which were eligible were omitted. What is more, Wang et al. did not investigate the association of AMD and mortality according to study characteristics such as types of AMD. We found a significant association between early AMD and risk of allcause mortality incidence.

There are several strengths in our study. A major advantage of the meta-analysis is that the present findings are based on cohort studies. Thus, this minimizes the possibility of recall and selection biases. Compared with the previous 


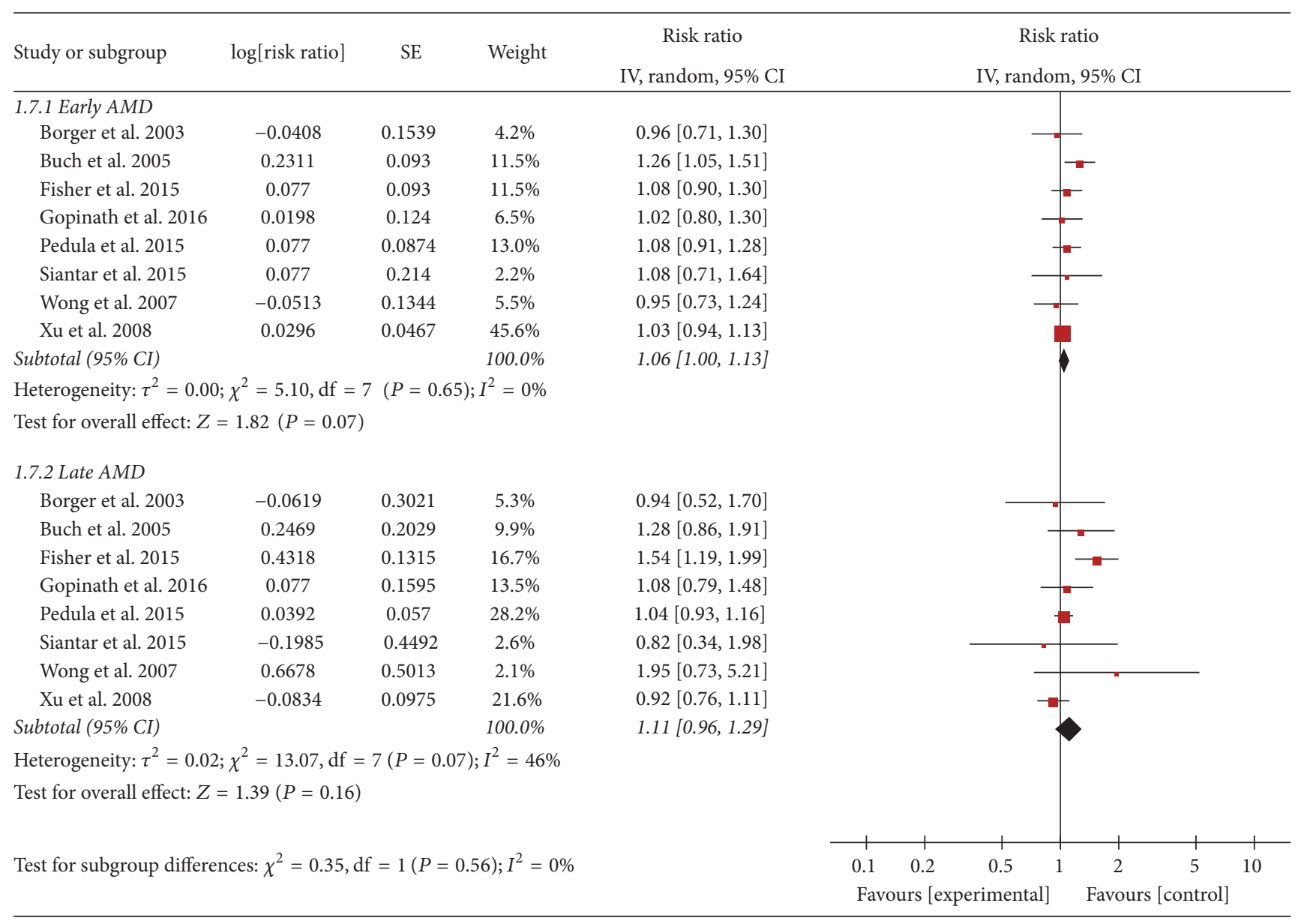

FIGURE 3: Adjusted relative risks of all-cause mortality associated with different AMD.

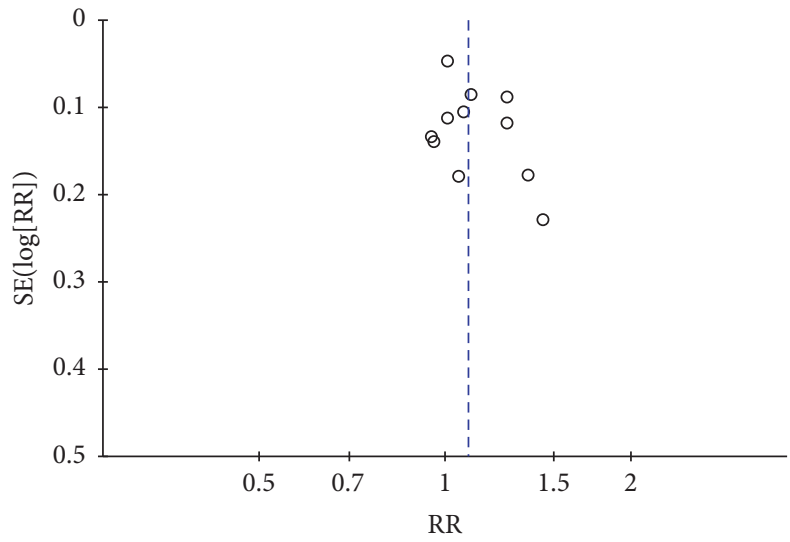

FIGURE 4: Funnel plots for the detection of public bias among studies that evaluated the associations of AMD with the risk of mortality.

meta-analysis, the risk estimates reported in the present study were a bit bigger. However, with accumulating evidence and enlarged sample size, we have enhanced statistical power to provide more precise and reliable risk estimates relating between AMD and all-cause mortality.
Limitations also of this meta-analysis should be acknowledged. First, the methods of AMD assessment varied across studies: four studies by ICD code and four studies by selfreport from study participants. This may lead to misclassification error and bias. Second, the meta-analysis was limited to English publications, and the possibility of unpublished reports was not yet identified. Third, residual confounding is still possible given that many studies did not adjust for several important potential factors in their models such as physical activity, smoking, unhealthy lifestyle factors, depression, and stress which are also risk factors for poor health outcomes in AMD patients.

\section{Conclusions}

In conclusion, this meta-analysis provides compelling evidence that persons with AMD had a higher risk of mortality than persons without AMD.

\section{Conflicts of Interest}

The authors declare that they have no conflicts of interest regarding the publication of this paper. 


\section{Acknowledgments}

The authors would like to acknowledge Wenzhou City Hospital of Traditional Chinese Medicine and Western Medicine Combined for supporting the work in this study.

\section{References}

[1] D. Pascolini and S. P. Mariotti, "Global estimates of visual impairment: 2010," British Journal of Ophthalmology, vol. 96, no. 5, pp. 614-618, 2012.

[2] L. S. Lim, P. Mitchell, J. M. Seddon, F. G. Holz, and T. Y. Wong, "Age-related macular degeneration," The Lancet, vol. 379, no. 9827, pp. 1728-1738, 2012.

[3] W. L. Wong, X. Su, X. Li et al., "Global prevalence of age-related macular degeneration and disease burden projection for 2020 and 2040: a systematic review and meta-analysis," The Lancet Global Health, vol. 2, no. 2, pp. e106-e116, 2014.

[4] K. L. Pennington and M. M. DeAngelis, "Epidemiology of age-related macular degeneration (AMD): associations with cardiovascular disease phenotypes and lipid factors," Eye and vision, vol. 3, article 34, 2016.

[5] R. D. Jager, W. F. Mieler, and J. W. Miller, "Age-related macular degeneration," The New England Journal of Medicine, vol. 358, no. 24, pp. 2606-2617, 2008.

[6] A. R. Shah and L. V. Del Priore, "Progressive visual loss in subfoveal exudation in age-related macular degeneration: a meta-analysis using Lineweaver-Burke plots," American Journal of Ophthalmology, vol. 143, no. 1, pp. 83-89.e2, 2007.

[7] P. H. Borger, R. van Leeuwen, C. A. A. Hulsman et al., "Is there a direct association between age-related eye diseases and mortality? The Rotterdam Study," Ophthalmology, vol. 110, no. 7, pp. 1292-1296, 2003.

[8] H. Buch, T. Vinding, M. La Cour, G. B. Jensen, J. U. Prause, and N. V. Nielsen, "Age-related maculopathy: a risk indicator for poorer survival in women: the Copenhagen City Eye Study," Ophthalmology, vol. 112, no. 2, pp. 305-312, 2005.

[9] D. E. Fisher, F. Jonasson, G. Eiriksdottir et al., "Age-related macular degeneration and mortality in community-dwelling elders: the age, gene/environment susceptibility Reykjavik study," Ophthalmology, vol. 122, no. 2, pp. 382-390, 2015.

[10] B. Gopinath, G. Liew, G. Burlutsky, and P. Mitchell, "Agerelated macular degeneration and risk of total and causespecific mortality over 15 years," Maturitas, vol. 84, pp. 63-67, 2016.

[11] R. C. Khanna, G. V. S. Murthy, P. Giridhar et al., "Cataract, visual impairment and long-term mortality in a rural cohort in india: the Andhra Pradesh Eye Disease Study," PLoS ONE, vol. 8, no. 10, Article ID e78002, 2013.

[12] M. D. Knudtson, B. E. K. Klein, and R. Klein, "Age-related eye disease, visual impairment, and survival: the Beaver Dam Eye study," Archives of Ophthalmology, vol. 124, no. 2, pp. 243-249, 2006.

[13] C. A. McCarty, Nanjan M. B., and Taylor H. R., "Vision impairment predicts 5 year mortality," The British Journal of Ophthalmology, vol. 85, no. 3, pp. 322-326, 2001.

[14] K. L. Pedula, A. L. Coleman, F. Yu et al., "Age-related macular degeneration and mortality in older women: the study of osteoporotic fractures," Journal of the American Geriatrics Society, vol. 63, no. 5, pp. 910-917, 2015.
[15] R. G. Siantar, C.-Y. Cheng, C. M. Gemmy Cheung et al., "Impact of visual impairment and eye diseases on mortality: The Singapore Malay Eye Study (SiMES)," Scientific Reports, vol. 5, Article ID 16304, 2015.

[16] M. Thiagarajan, J. R. Evans, L. Smeeth, R. P. L. Wormald, and A. E. Fletcher, "Cause-specific visual impairment and mortality: results from a population-based study of older people in the United Kingdom," Archives of Ophthalmology, vol. 123, no. 10, pp. 1397-1403, 2005.

[17] T. Y. Wong, G. Tikellis, C. Sun, R. Klein, D. J. Couper, and A. R. Sharrett, "Age-related macular degeneration and risk of coronary heart disease: the Atherosclerosis Risk in Communities Study," Ophthalmology, vol. 114, no. 1, pp. 86-91, 2007.

[18] L. Xu, Y. B. Li, Y. X. Wang, and J. B. Jonas, "Age-related macular degeneration and mortality: the Beijing Eye Study," Ophthalmologica, vol. 222, no. 6, pp. 378-379, 2008.

[19] J. Wang, Y. Xue, S. Thapa, L. Wang, J. Tang, and K. Ji, "Relation between age-related macular degeneration and cardiovascular events and mortality: a systematic review and meta-analysis," BioMed Research International, vol. 2016, Article ID 8212063, 10 pages, 2016.

[20] J. Zhang and K. F. Yu, "What's the relative risk? A method of correcting the odds ratio in cohort studies," Journal of the American Medical Association, vol. 280, no. 19, pp. 1690-1691, 1998.

[21] J. P. Higgins, S. G. Thompson, J. J. Deeks, and D. G. Altman, "Measuring inconsistency in meta-analyses," BMJ, vol. 327, no. 7414, pp. 557-560, 2003.

[22] R. DerSimonian and N. Laird, "Meta-analysis in clinical trials revisited," Contemporary Clinical Trials, vol. 45, pp. 139-145, 2015.

[23] P.-C. Bürkner and P. Doebler, "Testing for publication bias in diagnostic meta-analysis: a simulation study," Statistics in Medicine, vol. 33, no. 18, pp. 3061-3077, 2014.

[24] C. M. Cheung and T. Y. Wong, "Is age-related macular degeneration a manifestation of systemic disease?" New. Journal of International Medicine, vol. 276, no. 2, pp. 140-153, 2014.

[25] J. Kulmala, P. Era, O. Pärssinen et al., "Lowered vision as a risk factor for injurious accidents in older people," Aging Clinical and Experimental Research, vol. 20, no. 1, pp. 25-30, 2008.

[26] J. Tolman, R. D. Hill, J. J. Kleinschmidt, and C. H. Gregg, "Psychosocial adaptation to visual impairment and its relationship to depressive affect in older adults with age-related macular degeneration," Gerontologist, vol. 45, no. 6, pp. 747-753, 2005. 


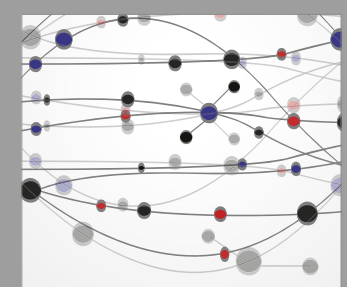

The Scientific World Journal
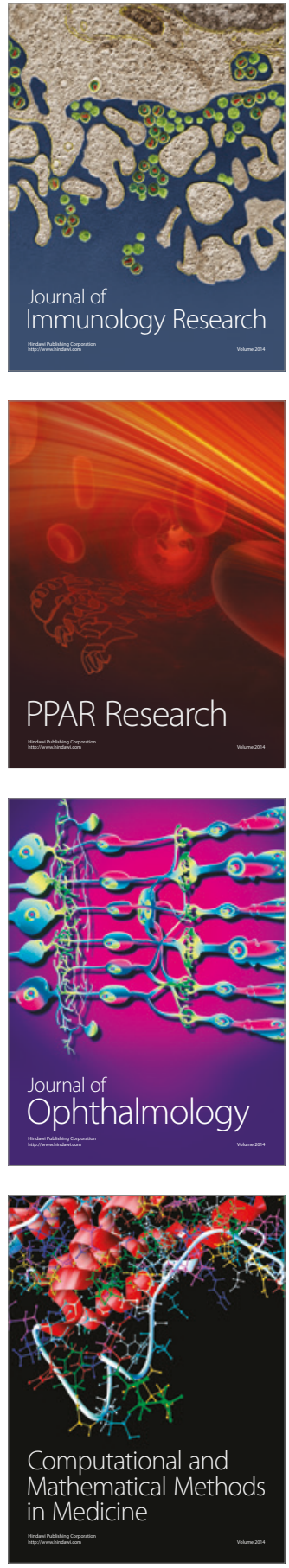

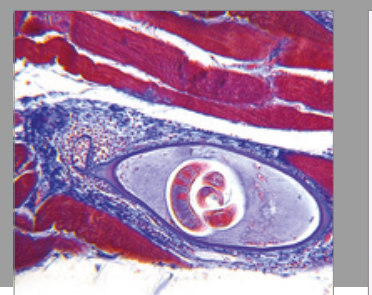

Gastroenterology Research and Practice
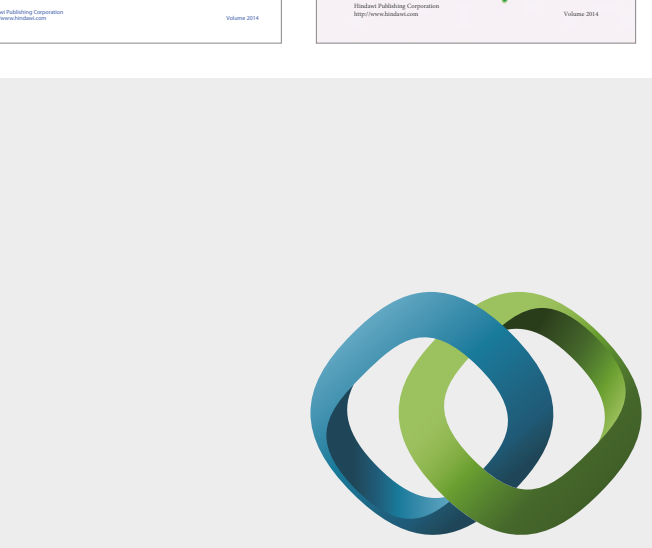

\section{Hindawi}

Submit your manuscripts at

https://www.hindawi.com
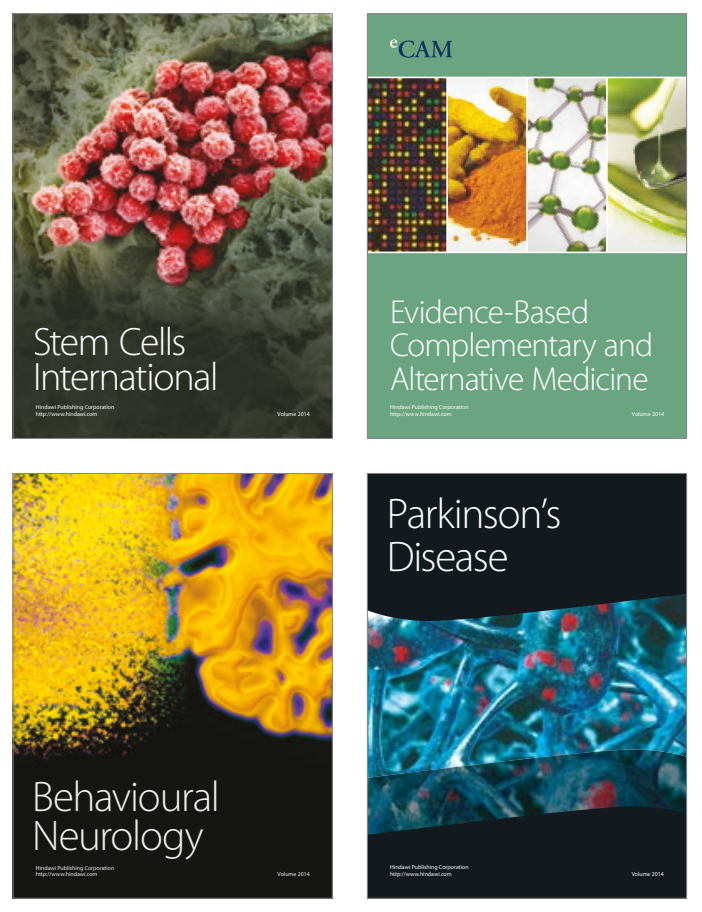
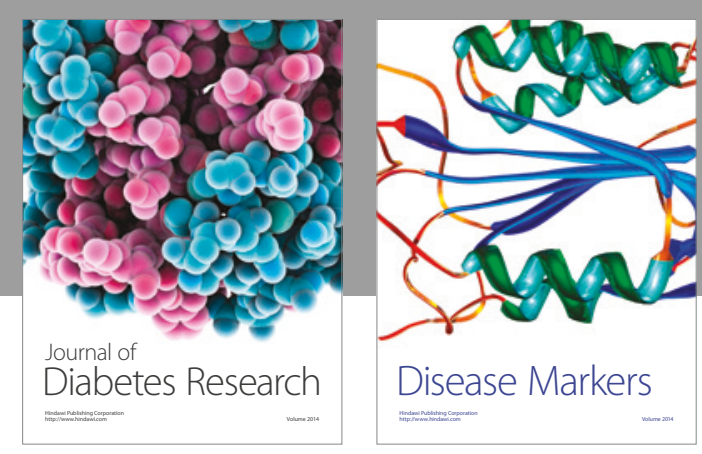

Disease Markers
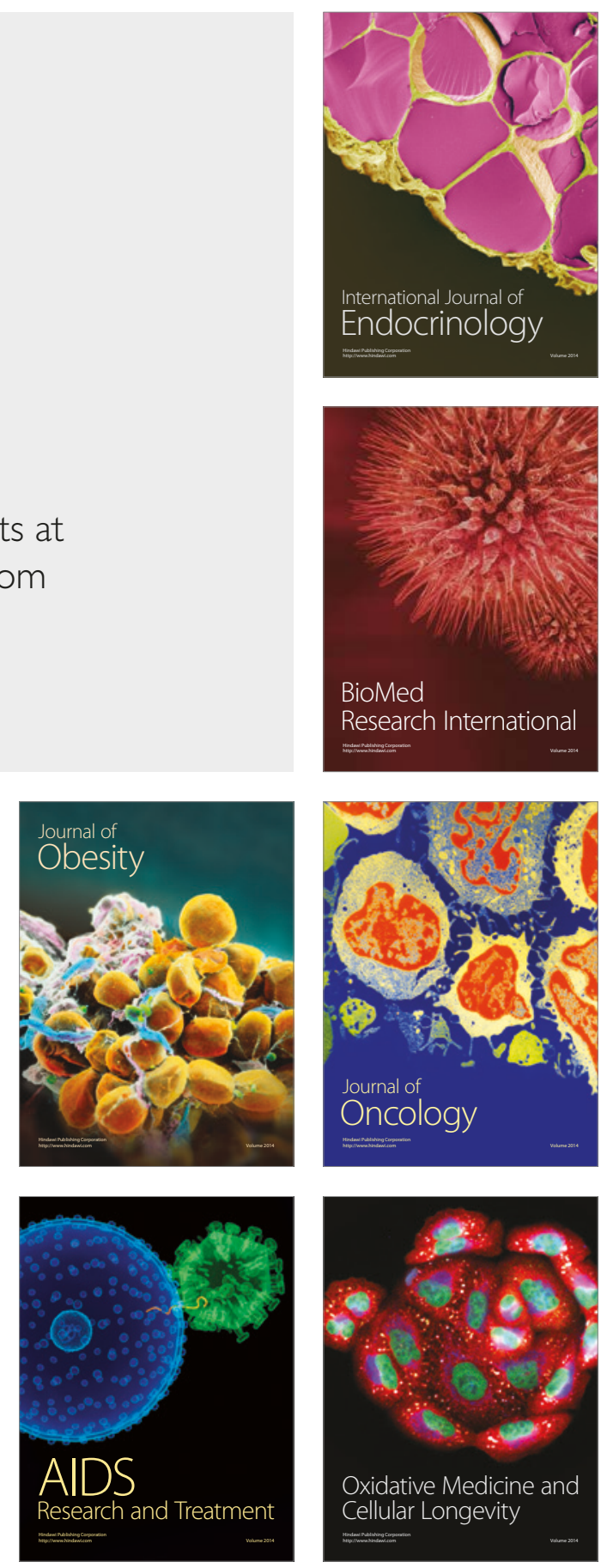